\title{
Nucleon-Nucleon Short-Range Correlations and Gribov Inelastic Shadowing in High Energy Hadron-Nucleus Collisions ${ }^{1}$
}

\author{
Claudio Ciofi degli Atti \\ Istituto Nazionale di Fisica Nucleare, Sezione di Perugia, Via A. Pascoli, I-06123 Perugia, Italy
}

\begin{abstract}
Different types of high-energy hadron-nucleus cross sections are discussed emphasizing the role played by Nucleon-Nucleon (NN) Short-Range Correlations (SRC) and Gribov Inelastic Shadowing (IS).
\end{abstract}

Keywords: short-range correlations, Gribov inelastic shadowing, high-energy collisions

PACS: $24.85 .+p, 13.85 . \mathrm{Lg}, 13.85 . \mathrm{Lg}, 25.55 . \mathrm{Ci}$

A large number of theoretical approaches aimed at describing hadron-nucleus collisions at multi $\mathrm{GeV}$ energies, are based upon Glauber multiple scattering theory [1] within the independent-particle model description of the nucleus. The latter approximation seems to be out of date, for nuclear constituents spend part of their time in strongly correlated configurations [2], as quantitatively demonstrated by recent experimental data [3] (see Fig. 1). Besides NN SRC, intermediate hadron-hadron inelastic scattering (Gribov IS [4]), lacking in the Glauber approach, should be taken into account. As a matter of fact, the importance of the effects of both SRC and Gribov IS in different high energy scattering processes have been studied in a series of recent papers [5]-[10], finding that the two effects act frequently in the opposite directions. The nuclear quantity entering most Glauber-like calculations is the modulus squared of the nuclear wave function $\left|\psi_{0}\right|^{2}$, whose exact expansion [1] is usually approximated by the lowest order, fully uncorrelated term, viz

$$
\left|\psi_{0}\left(\mathbf{r}_{1}, \ldots, \mathbf{r}_{A}\right)\right|^{2}=\prod_{j=1}^{A} \rho_{1}\left(\mathbf{r}_{j}\right)+\sum_{i<j} \Delta\left(\mathbf{r}_{i}, \mathbf{r}_{j}\right) \prod_{k \neq i, j} \rho_{1}\left(\mathbf{r}_{k}\right)+\ldots . \simeq \prod_{j=1}^{A} \rho_{1}\left(\mathbf{r}_{j}\right) .
$$

Here the two-body contraction $\Delta\left(\mathbf{r}_{i}, \mathbf{r}_{j}\right)=\rho_{2}\left(\mathbf{r}_{i}, \mathbf{r}_{j}\right)-\rho_{1}\left(\mathbf{r}_{i}\right) \rho_{1}\left(\mathbf{r}_{j}\right)$, contains the effect of SRC, leading to a hole in the two-body density $\rho_{2}$ at short inter-nucleon separations $r=\left|\mathbf{r}_{i}-\mathbf{r}_{j}\right|$ (see Fig. 2). SRC generate an additional contribution to the nuclear thickness function as follows $\left(\mathbf{r}_{i}=\left\{\mathbf{s}_{i}, z_{i}\right\}\right)[5]$

$$
\Delta T_{A}^{h}(b)=\frac{1}{\sigma_{\text {tot }}^{h N}} \int d^{2} \mathbf{s}_{1} d^{2} \mathbf{s}_{2} \Delta_{A}^{\perp}\left(\mathbf{s}_{1}, \mathbf{s}_{2}\right) \operatorname{Re} \Gamma^{p N}\left(\mathbf{b}-\mathbf{s}_{1}\right) \operatorname{Re} \Gamma^{p N}\left(\mathbf{b}-\mathbf{s}_{2}\right),
$$

\footnotetext{
1 Talk given at DIFFRACTION 2012, International Workshop on Diffraction in High-Energy Physics, Lanzarote, Canary Islands (Spain), September 10-15, 2012.
} 


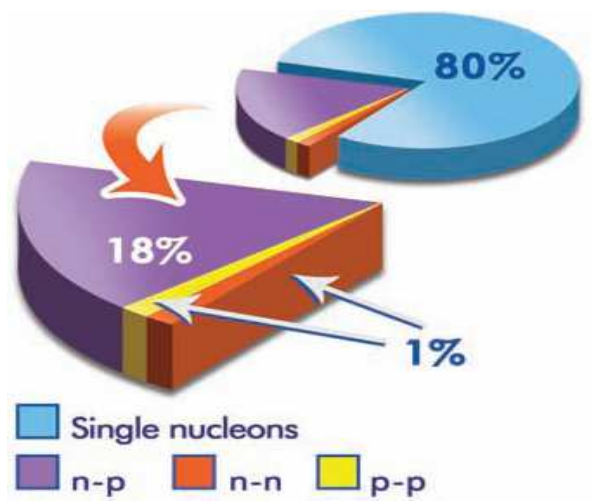

FIGURE 1. The percentage of NN SRC found experimentally in ${ }^{12} C$ nucleus (after Ref. [3]).
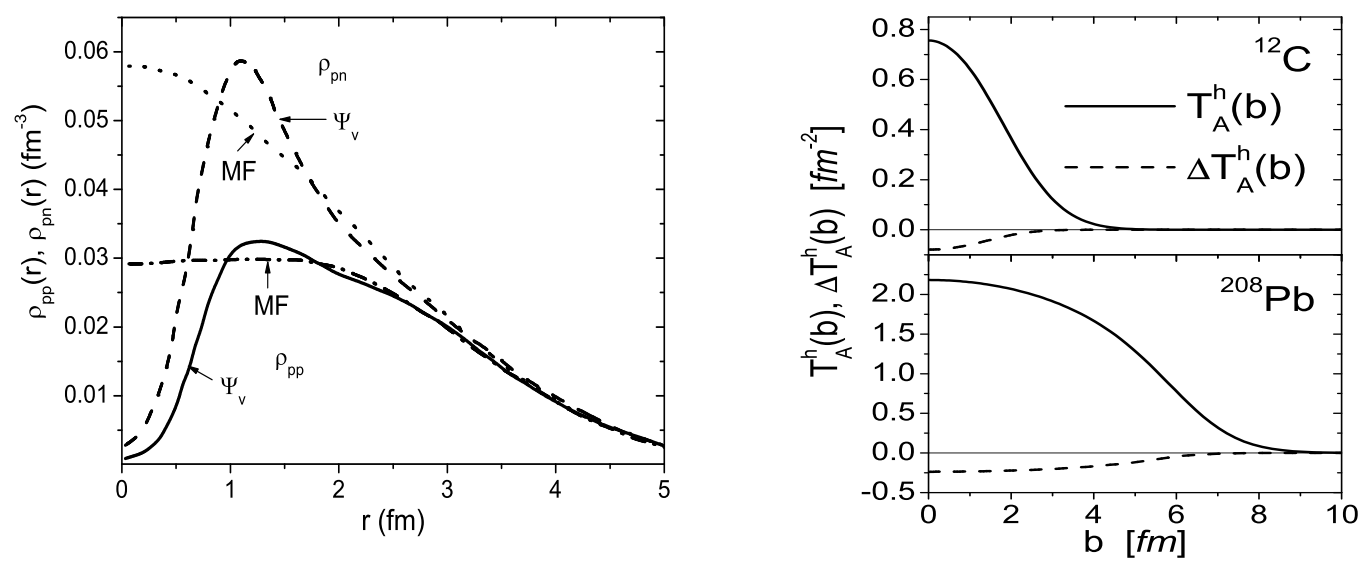

FIGURE 2. (Left) The proton-neutron $(p n)$ and proton-proton $(p p)$ two-body densities $\rho_{2} \equiv \rho_{N N}$ in ${ }^{16} \mathrm{O}$ calculated [2] within a mean field model (MF) and by solving the many-body problem with a realistic NN interaction $\left(\Psi_{v}\right)$ (after Ref. [2]). (Right) The thickness function $T_{A}^{h}(b)$ and the correlation contribution, $\Delta T_{A}^{h}(b)$, in $p-{ }^{12} C$ and $p-{ }^{208} P b$ collisions at HERA-B energies. The total thickness function is given by $\widetilde{T}_{A}^{h}=T_{A}^{h}-\Delta T_{A}^{h}$ (after Ref. [6]).

where $\Delta_{A}^{\perp}\left(\mathbf{s}_{1}, \mathbf{s}_{2}\right)$ is the transverse contraction and the total thickness function is $\widetilde{T}_{A}^{h}=$ $T_{A}^{h}-\Delta T_{A}^{h}$. The thickness functions of ${ }^{12} C$ and ${ }^{208} P b$ at HERA-B energies are given in Fig. 2. In Ref. [5], it has been shown that SRC increase the total neutron-nucleus cross section at high energies, making the nucleus more opaque, unlike Gribov IS corrections (considered in Ref. [5] in lowest order) which increase nuclear transparency (see Fig. 3). An exhaustive calculation [6] of the total, $\sigma_{t o t}^{h A}$, elastic, $\sigma_{e l}^{h A}$, quasi-elastic, $\sigma_{q e l}^{h A}$, inelastic, $\sigma_{i n}^{h A}$, and diffractive dissociation cross sections, which include both SRC and Gribov IS, summed to all orders by the dipole approach [7, 8, 9], confirms the opposite roles played by SRC and IS (Table 1). The thickness function due to SRC and Gribov IS, which depends also upon the dipole transverse dimensions $\mathbf{r}_{T}$, the light cone variable $\alpha$, and 


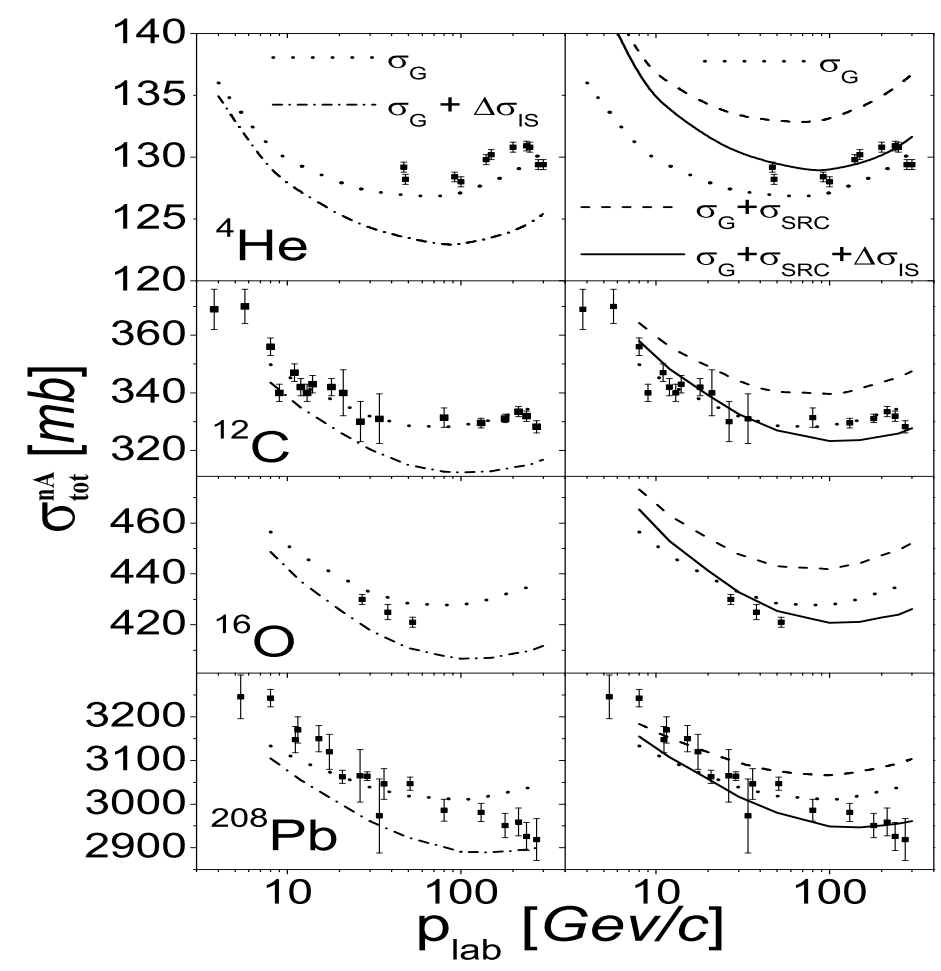

FIGURE 3. The total neutron-nucleus cross section $\sigma_{t o t}^{n A} v s p_{\text {lab. }}$ Left panel: Glauber single density approximation $\left(\sigma_{G} ;\right.$ dots $)$ and Glauber plus Gribov inelastic shadowing $\left(\sigma_{G}+\Delta \sigma_{I S}\right.$; dot-dash $)$. Right panel: Glauber $\left(\sigma_{G}\right.$; dots); Glauber plus SRC $\left(\sigma_{G}+\sigma_{S R C}\right.$; dashes $)$; Glauber plus SRC plus Gribov inelastic shadowing $\left(\sigma_{G}+\sigma_{S R C}+\Delta \sigma_{I S} ;\right.$ full $)$ (after Ref. [5]).

TABLE 1. Various $p-208 P b$ cross sections at LHC energies (after Ref. [6]).

\begin{tabular}{c|cccc}
\hline \hline${ }^{208} \mathrm{~Pb}$ & Glauber & $\begin{array}{c}\text { Glauber } \\
+ \text { SRC }\end{array}$ & $\begin{array}{c}\text { q-2q model } \\
\text { +SRC }\end{array}$ & $\begin{array}{c}\text { 3q model } \\
\text { +SRC }\end{array}$ \\
\hline$\sigma_{\text {tot }}^{N A}[\mathrm{mb}]$ & 3850.63 & 3885.77 & 3833.26 & 3839.26 \\
\hline$\sigma_{e l}^{N A}[\mathrm{mb}]$ & 1664.76 & 1690.48 & 1655.70 & 1660.67 \\
\hline$\sigma_{q e}^{N A}[\mathrm{mb}]$ & 120.92 & 112.65 & 113.37 & 113.88 \\
\hline
\end{tabular}

the dipole-nucleon cross section and profile function has the form

$$
\begin{aligned}
& \Delta T_{A}^{d i p}\left(b, \mathbf{r}_{T}, \alpha\right)= \\
& =\frac{1}{\sigma_{d i p}\left(r_{T}\right)} \int d^{2} \mathbf{s}_{1} d^{2} \mathbf{s}_{2} \Delta_{A}^{\perp}\left(\mathbf{s}_{1}, \mathbf{s}_{2}\right) \operatorname{Re} \Gamma^{\bar{q} q, N}\left(\mathbf{b}-\mathbf{s}_{1}, \mathbf{r}_{T}, \alpha\right) \operatorname{Re} \Gamma^{\bar{q} q, N}\left(\mathbf{b}-\mathbf{s}_{2}, \mathbf{r}_{T}, \alpha\right) .(3)
\end{aligned}
$$

SRC and Gribov IS affect also the number of inelastic collisions $N_{\text {coll }}=A \sigma_{i n}^{h N} / \sigma_{i n}^{h A}$ which is the normalization factor used to obtain the nucleus to nucleon ratio of the 
cross section of a hard reaction. The results of calculations $[6,10]$ based upon realistic one- and two-body densities and correlation functions [11], are shown in Table 2. The behavior of $N_{\text {coll }}$ is entirely governed by the non-diffractive $\sigma_{i n}^{N A}$ which is decreased by SRC and increased by Gribov IS.

TABLE 2. Number of inelastic collisions $N_{c o l l}$ in $p-{ }^{208} \mathrm{~Pb}$ scattering at RHIC and LHC energies (after Ref. [10]).

\begin{tabular}{c|cccccc}
\hline \hline & \multicolumn{7}{c}{ GLAUBER } \\
\hline & $\sigma_{i n}^{N N}[\mathrm{mb}]$ & $\sigma_{\text {tot }}^{N A}[\mathrm{mb}]$ & $\sigma_{e l}^{N A}[\mathrm{mb}]$ & $\sigma_{q e l}^{N A}[\mathrm{mb}]$ & $\sigma_{\text {in }}^{N A}[\mathrm{mb}]$ & $N_{\text {coll }}$ \\
\hline RHIC & 42.10 & 3297.56 & 1368.36 & 66.06 & 1863.14 & 4.70 \\
\hline LHC & 68.30 & 3850.63 & 1664.76 & 120.92 & 2064.95 & 6.88 \\
\hline \hline & \multicolumn{7}{c}{ GLAUBER+SRC } \\
\hline & $\sigma_{i n}^{N N}[\mathrm{mb}]$ & $\sigma_{\text {tot }}^{N A}[\mathrm{mb}]$ & $\sigma_{e l}^{N A}[\mathrm{mb}]$ & $\sigma_{q e l}^{N A}[\mathrm{mb}]$ & $\sigma_{\text {in }}^{N A}[\mathrm{mb}]$ & $N_{\text {coll }}$ \\
\hline RHIC & 42.10 & 3337.57 & 1398.08 & 58.47 & 1881.02 & 4.65 \\
\hline LHC & 68.30 & 3885.77 & 1690.48 & 112.65 & 2082.64 & 6.82 \\
\hline \hline & & & GLAUBER+SRC+GRIBOV $(q-2 q)$ & & \\
\hline & $\sigma_{i n}^{N N}[\mathrm{mb}]$ & $\sigma_{\text {tot }}^{N A}[\mathrm{mb}]$ & $\sigma_{e l}^{N A}[\mathrm{mb}]$ & $\sigma_{q e l}^{N A}[\mathrm{mb}]$ & $\sigma_{\text {in }}^{N A}[\mathrm{mb}]$ & $N_{\text {coll }}$ \\
\hline RHIC & 42.10 & 3228.11 & 1314.04 & 71.99 & 1842.08 & 4.75 \\
\hline LHC & 68.30 & 3833.26 & 1655.70 & 113.37 & 2064.19 & 6.88 \\
\hline \hline
\end{tabular}

\section{ACKNOWLEDGMENT}

I am indebted to Boris Kopeliovich, Irina Potashnikova and Ivan Schmidt, Universidad Federico Santa María, Valparaíso, Chile, and to Massimiliano Alvioli and Chiara Benedetta Mezzetti, INFN, Perugia, Italy, for a fruitful collaboration.

\section{REFERENCES}

1. R. J. Glauber, in Lectures in Theoretical Physics, W. E. Brittin et al Editors, New York (1959).

2. S. C. Pieper, R. B. Wiringa, V. R. Pandharipande, Phys. Rev. C46 (1992) 1741.

3. R. Subedi, R. Shneor, P. Monaghan, B. D. Anderson, et al., Probing Cold Dense Nuclear Matter, Science 320, 1476 (2008) [nucl-ex 0908.1514].

4. V. N. Gribov, Sov. JETP 29 (1969) 483.

5. M. Alvioli, C. Ciofi degli Atti, I. Marchino, V. Palli, H. Morita Phys. Rev.C78 (2008) 031601(R).

6. M. Alvioli, C. Ciofi degli Atti, B. Z. Kopeliovich, I. K. Potashnikova, I. Schmidt, Phys. Rev. C81 (2010) 025204.

7. B. Z. Kopeliovich, L. I. Lapidus, A. B. Zamolodchikov, JETP Lett. 33 (1981) 595. [Pisma Zh. Eksp. Teor. Fiz. 33 (1981) 595].

8. B. Z. Kopeliovich, Phys. Rev. C68 (2003) 044906.

9. B. Z. Kopeliovich, I. K. Potashnikova and I. Schmidt Phys. Rev. C73 (2006) 034901.

10. C. Ciofi degli Atti, C. B. Mezzetti, B. Z. Kopeliovich, I. K. Potashnikova, I. Schmidt, Phys. Rev. C84 (2011) 025205 [nucl-th 1105.1080v2].

11. M. Alvioli, C. Ciofi degli Atti, H. Morita, Phys. Rev. Lett. 100 (2008) 162503. 